\title{
Correction to: The efficacy and safety of menatetrenone in the management of osteoporosis: a systematic review and meta-analysis of randomized controlled trials
}

\author{
S. Su ${ }^{1,2} \cdot$ N. He ${ }^{1,2} \cdot$ P. Men ${ }^{1} \cdot$ C. Song ${ }^{3} \cdot$ S. Zhai ${ }^{1}$ \\ Published online: 27 August 2021 \\ (C) International Osteoporosis Foundation and National Osteoporosis Foundation 2021
}

\section{Correction to: Osteoporosis International (2019) 30:1175-1186}

https://doi.org/10.1007/s00198-019-04853-7

The original version of this article, published on February 7 , 2019, contained a mistake.

The correct information is given below. The original article has been corrected.

Results - Efficacy evaluations - Menatetrenone versus placebo/no additional drug:

"In addition, analysis of four studies [22,24,26,38] $(\mathrm{N}=\mathbf{3 5 6})$ revealed a significantly greater percent increase in lumbar BMD from baseline for menatetrenone compared with placebo or no additional anti-osteoporotic drug ( $\mathrm{MD}=\mathbf{2 . 0 2 \%}$, 95\% CI 1.05 to $2.99 \%, P<\mathbf{0 . 0 0 0 1}$ ) (Fig. 3 and Online Resource 3)."

Table 1. Characteristics of the included RCTs

In the "Outcome" column, the Orimo 1999 study should be "9,12,13" rather than " $5,9,12,13$ ".

\section{Correction in supplementary material}

The online version of the original article can be found at https://oi.org/ 10.1007/s00198-019-04853-7

\section{S. Zhai}

zhaisuodi@163.com

1 Department of Pharmacy, Peking University Third Hospital, 49 Huayuan North Road, Haidian District, Beijing 100191, China

2 Department of Pharmacy Administration and Clinical Pharmacy, School of Pharmaceutical Science, Peking University, Beijing, China

3 Department of Orthopaedics, Peking University Third Hospital, Beijing, China 
Study

ID
No. of No. of

MD $(95 \% \mathrm{Cl}) \quad$ studies patients $\quad$-squared(\%)
ucOC $(\mathrm{ng} / \mathrm{ml})$

ucOC/OC(\%)

LBMD change $(\mathrm{g} / \mathrm{cm} 2$

LBMD change(\%)

vs. calcium

ucOC (ng/ml)

LBMD change(\%)

vs. alfacalcidol

LBMD change $(\mathrm{g} / \mathrm{cm} 2)$

LBMD change(\%)

$\begin{array}{llll}-1.17(-2.82,0.49) 3 & 2038 & 85 \\ -0.22(-0.34,-0.10) 2 & 75 & 0 \\ 0.05(0.01,0.09) \quad 5 & 658 & 78 \\ 2.02(1.05,2.99) & 4 & 356 & 35\end{array}$

$-2.00(-3.38,-0.62) 1 \quad 109 \quad N A$

$-0.20(-1.59,1.19) 1 \quad 109 \quad$ NA

$0.00(-0.03,0.03) 2 \quad 275 \quad 0$

$1.70(-5.17,8.57) \quad 1 \quad 61 \quad N A$

Fig. 3 Summary of results of the meta-analyses for continuous variables (only summary effect estimate of menatetrenone vs. placebo - LBMD change (\%) was changed)

Publisher's note Springer Nature remains neutral with regard to jurisdictional claims in published maps and institutional affiliations. 